\title{
Stability Analysis of a Class of Second Order Sliding Mode Control Including Delay in Input
}

\author{
Pedro R. Acosta \\ Instituto Tecnológico de Chihuahua, Avenida Tecnológico 2909, 31310 Chihuahua, CHIH, Mexico \\ Correspondence should be addressed to Pedro R. Acosta; pacosta@itchihuahua.edu.mx
}

Received 27 July 2013; Revised 4 October 2013; Accepted 6 October 2013

Academic Editor: Xudong Zhao

Copyright ( 2013 Pedro R. Acosta. This is an open access article distributed under the Creative Commons Attribution License, which permits unrestricted use, distribution, and reproduction in any medium, provided the original work is properly cited.

\begin{abstract}
This paper deals with a class of second order sliding mode systems. Based on the derivative of the sliding surface, sufficient conditions are given for stability. However, the discontinuous control signal depend neither on the derivative of sliding surface nor on its estimate. Time delay in control input is also an important issue in sliding mode control for engineering applications. Therefore, also sufficient conditions are given for the time delay size on the discontinuous input signal, so that this class of second order sliding mode systems might have amplitude bounded oscillations. Moreover, amplitude of such oscillations may be estimated. Some numerical examples are given to validate the results. At the end, some conclusions are given on the possibilities of the results as well as their limitations.
\end{abstract}

\section{Introduction}

Sliding mode control has been effectively used in engineering for more than three decades. One of the major concerns for some applications is chattering in output or control signal. Along with other techniques, higher order sliding modes have been proposed to alleviate chattering. Most used algorithms for high sliding motion are the twisting controller [1], suboptimal controller [2], and the super twisting controller [3]. These algorithms need to measure or estimate the derivative of the sliding surface. The super twisting algorithm is used to get robust differentiators, so that it can be used together with the other algorithms to get such a derivative. Recently [4], homogeneity has been used to ease parameter design for second order sliding mode controllers. Some recent examples of engineering applications using second order sliding mode controllers are [5-8].

When delays arise, sliding mode controller performance deteriorates. Such delays may occur in states and/or inputs and have been treated using several approaches (see e.g., [9-16]). In [12], a good review is given for different control techniques up to 2003. When only a delayed measure is available for the control signal, two techniques have been used to compensate delay effects: predictors and integral sliding mode control. Some instances are $[11,12,14]$, respectively.
On the other hand, concerning analysis for existence and amplitude of steady oscillations due to delays, very few works have been reported, such as [17], where sufficient conditions are given for steady oscillations to exist in a first order sliding mode system; describing function and Poincaré map approach are used in [18] to analyze oscillations for a system with hysteresis. In [19], a different approach for perturbed first sliding mode systems is reported. For second order sliding mode systems with delay, [20] reported sufficient conditions for steady oscillations to occur in a class of systems; in [21], an analysis is given about oscillations for the suboptimal algorithm considering delay in the input.

Motivated by the aforementioned analysis, this paper presents sufficient stability conditions for a class of sliding mode system that does not require a measure of the derivative of the sliding surface in order to provide the control input. Also, sufficient conditions are given for stable oscillations to exist if a delay occurs in the input, as well as an estimate for their amplitude. The class of systems treated here is a more general class of systems than the one reported in [20] and using a different approach. In Section 2, the problem is formulated. Section 3 presents the main results by way of two propositions and their proofs, giving sufficient conditions for finite time sliding motion stability without delay and stability 
for oscillations when delay in input occurs. Next, in Section 4, some numerical examples are given and conclusions for the reported results are related.

\section{Problem Statement}

Let us consider a second order sliding mode system in the form

$$
\begin{gathered}
\dot{x}_{1}=x_{2}, \\
\dot{x}_{2}=f\left(t, x_{1}, x_{2}\right)-k \operatorname{sign}\left(x_{1}(t)\right),
\end{gathered}
$$

where $f\left(t, x_{1}, x_{2}\right)$ is smooth and $k>0$. The first issue to consider is getting sufficient conditions such that the system has a stable equilibrium point in $x_{1}=0, x_{2}=0$ and reached in finite time, considering a domain of attraction $D$

$$
\mathbf{x}=\left[\begin{array}{ll}
x_{1} & x_{2}
\end{array}\right]^{T} \in D .
$$

Now, if conditions for stability are met for (1), when a delay is present in the control input as follows:

$$
\begin{gathered}
\dot{x}_{1}=x_{2}, \\
\dot{x}_{2}=f\left(t, x_{1}, x_{2}\right)-k \operatorname{sign}\left(x_{1}(t-h)\right),
\end{gathered}
$$

with $\mathbf{x} \in D, \forall t \in[-h, 0), x_{1}$ might have steady oscillations if the time delay is sufficiently small, such that the system remains in the attraction domain. Otherwise, its dynamics will be unstable. Hence, it is important to get sufficient conditions for delay size, such that the system remains in the domain of attraction and an estimate for the amplitude of the oscillations. Next section shows sufficient conditions to reach the surface in finite time without delay and for existence of bounded amplitude oscillations in presence of delay.

\section{Sufficient Conditions for Second Order Sliding Modes without Delay and Amplitude Bounded Oscillations with Delay}

In the following proposition, sufficient conditions are given for finite time reaching and, stability of the equilibrium point $x_{1}=0, x_{2}=0$ in system (1) considering a domain of attraction.

Proposition 1. Consider system (1) with $f(t, 0,0)=0$. If $k-\left|f\left(t, x_{1}, x_{2}\right)\right| \geq c_{0}>0$ and, moreover, after $x_{1}$ first zero crossing, the following is met thereafter:

$$
\left|\frac{c_{n}}{c_{n+1}}\right|>\frac{k-\left|f_{n \max }\left(t, x_{1}, x_{2}\right)\right|}{k-\left|f_{n+1 \max }\left(t, x_{1}, x_{2}\right)\right|},
$$

where $t_{n}$ is the nth zero crossing time, $c_{n}$ and $c_{n+1}$ are the values for $x_{2}\left(t_{n}\right)$ and $x_{2}\left(t_{n+1}\right)$ in the $n$th and $n+1$ th zero crossing time for $x_{1}$. Also, $\left|f_{n \text { max }}\left(t, x_{1}, x_{2}\right)\right|$ is the biggest value of $\left|f\left(t, x_{1}, x_{2}\right)\right|$ in the interval $t_{n} \leq t<t_{n+1}$ and $\left|f_{n+1 \max }\left(t, x_{1}, x_{2}\right)\right|$ is the biggest value of $\left|f\left(t, x_{1}, x_{2}\right)\right|$ in the interval $t_{n+1} \leq t<t_{n+2}$. Then, system (1) has a stable equilibrium point in $x_{1}=0, x_{2}=0$ which will be reached in finite time.
Proof. As a first step, let us demonstrate that there exists a finite time for $x_{1}(t)$ zero crossing. Without loss of generality, let us consider $x_{1}(0)>0$, therefore, in a neighborhood $t^{+}$of $t=0$ we will have

$$
\begin{gathered}
\dot{x}_{1}=x_{2}, \\
\dot{x}_{2}=f\left(t, x_{1}, x_{2}\right)-k .
\end{gathered}
$$

From hypothesis, $k-\left|f\left(0, x_{1}(0), x_{2}(0)\right)\right| \geq c_{0}>0$ and hence

$$
\dot{x}_{2} \leq-c_{0} \text {. }
$$

Therefore,

$$
x_{2}(t) \leq-c_{0} t+x_{2}(0)
$$

And so

$$
x_{1}(t) \leq-\frac{c_{0} t^{2}}{2}+x_{2}(0) t+x_{1}(0) \text {. }
$$

Hence, in a finite time, $t_{1}, x_{1}\left(t_{1}\right)=0$, where

$$
t_{1} \leq \frac{x_{2}(0)+\sqrt{x_{2}^{2}(0)+2 c_{0} x_{1}(0)}}{c_{0}} .
$$

Now, as a second step, let us demonstrate by induction that in a finite time the equilibrium point is reached. Without loss of generality, assume that the $n$th $x_{1}(t)$ zero crossing goes from positive to negative, and therefore $x_{2}\left(t_{n}\right)=c_{n}$ with $c_{n}<0$. Hence, for $t_{n} \leq t \leq t_{n+1}$, where $t=t_{n+1}$ is the next time for zero crossing, we will have

$$
\begin{gathered}
\dot{x}_{1}=x_{2}, \\
\dot{x}_{2}=f\left(t, x_{1}, x_{2}\right)+k .
\end{gathered}
$$

And therefore

$$
x_{2}(t) \geq\left(k-\left|f_{n \max }\left(t, x_{1}, x_{2}\right)\right|\right)\left(t-t_{n}\right)+c_{n} .
$$

So that

$$
x_{1}(t) \geq \frac{\left(k-\left|f_{n \max }\left(t, x_{1}, x_{2}\right)\right|\right)\left(t-t_{n}\right)^{2}}{2}+c_{n}\left(t-t_{n}\right) .
$$

From the above, for the following zero crossing, $x_{1}\left(t_{n+1}\right)=0$,

$$
\left(t_{n+1}-t_{n}\right) \leq \frac{-2 c_{n}}{\left(k-\left|f_{n \max }\left(t, x_{1}, x_{2}\right)\right|\right)} .
$$

To assure finite time for equilibrium point reaching, it only has to be guaranteed that $\left(t_{n+1}-t_{n}\right)>\left(t_{n+2}-t_{n+1}\right)$ since if $\left(t_{n+1}-t_{n}\right)=T$ and hence $\left(t_{n+2}-t_{n+1}\right) \leq a_{n} T$, where $0<a_{n} \leq$ $a<1$, total time will be

$$
\begin{aligned}
\sum_{n=1}^{\infty}\left(t_{n+1}-t_{n}\right) & \leq T+a T+a^{2} T+\cdots=T \sum_{n=1}^{\infty} a^{n-1} \\
& =T \frac{1}{1-a} .
\end{aligned}
$$


From (13), and a similar development for negative to positive zero crossing, the following can be deduced:

$$
\begin{aligned}
\left(t_{n+2}-t_{n+1}\right)+\left(t_{n+1}-t_{n}\right) \leq & \frac{\left|2 c_{n+1}\right|}{\left(k-\left|f_{n+1 \max }\left(t, x_{1}, x_{2}\right)\right|\right)} \\
& +\frac{\left|2 c_{n}\right|}{\left(k-\left|f_{n \max }\left(t, x_{1}, x_{2}\right)\right|\right)} .
\end{aligned}
$$

Now, considering that to comply with the supposed sufficient condition for reaching the equilibrium point in finite time $\left(t_{n+1}-t_{n}\right)=T$ and $\left(t_{n+2}-t_{n+1}\right) \leq a_{n} T$, the following must be complied as well:

$$
\begin{aligned}
\left(1+a_{n}\right)\left(t_{n+1}-t_{n}\right) \leq & \frac{\left|2 c_{n+1}\right|}{\left(k-\left|f_{n+1 \max }\left(t, x_{1}, x_{2}\right)\right|\right)} \\
& +\frac{\left|2 c_{n}\right|}{\left(k-\left|f_{n \max }\left(t, x_{1}, x_{2}\right)\right|\right)} .
\end{aligned}
$$

So that, making

$$
\begin{aligned}
& \frac{1}{\left(t_{n+1}-t_{n}\right)} \\
& \quad \times\left[\frac{\left|2 c_{n+1}\right|}{\left(k-\left|f_{n+1 \max }\left(t, x_{1}, x_{2}\right)\right|\right)}+\frac{\left|2 c_{n}\right|}{\left(k-\left|f_{n \max }\left(t, x_{1}, x_{2}\right)\right|\right)}\right] \\
& <2,
\end{aligned}
$$

it is guaranteed that $1+a_{n}<2$.

Now, using again (13) together with (17)

$$
\begin{aligned}
& \frac{\left|c_{n+1}\right|}{\left(k-\left|f_{n+1 \max }\left(t, x_{1}, x_{2}\right)\right|\right)}+\frac{\left|c_{n}\right|}{\left(k-\left|f_{n \max }\left(t, x_{1}, x_{2}\right)\right|\right)} \\
& <\frac{\left|2 c_{n}\right|}{\left(k-\left|f_{n \max }\left(t, x_{1}, x_{2}\right)\right|\right)} .
\end{aligned}
$$

Therefore, it is sufficient

$$
\frac{\left|c_{n+1}\right|}{\left(k-\left|f_{n+1 \max }\left(t, x_{1}, x_{2}\right)\right|\right)}<\frac{\left|c_{n}\right|}{\left(k-\left|f_{n \max }\left(t, x_{1}, x_{2}\right)\right|\right)} .
$$

In other words

$$
\frac{\left|c_{n}\right|}{\left|c_{n+1}\right|}>\frac{\left(k-\left|f_{n \max }\left(t, x_{1}, x_{2}\right)\right|\right)}{\left(k-\left|f_{n+1 \max }\left(t, x_{1}, x_{2}\right)\right|\right)} \text {. }
$$

Remark 2. If $\left|f_{n \text { max }}\left(t, x_{1}, x_{2}\right)\right|$ and $\left|f_{n+1 \text { max }}\left(t, x_{1}, x_{2}\right)\right|$ are not known, instead of (20) we may use as a more conservative condition $k-\left|f\left(t, x_{1}, x_{2}\right)\right| \geq c_{0}$, together with

$$
\frac{\left|c_{n}\right|}{\left|c_{n+1}\right|}>1
$$

When a delay exists in the discontinuous control signal, the equilibrium point cannot be reached. If the dynamics are in the domain of attraction, amplitude bounded oscillations are obtained. Next proposition presents sufficient conditions for an upper bound on the delay in order to get amplitude bounded oscillations.

Proposition 3. Considering system (1), complying with conditions of Proposition 1, a sufficient condition such that system (3) has steady oscillations is

$$
h \leq \min \left\{\frac{M_{x_{1}}^{\prime+}}{M_{x_{2}}^{\prime+}}, \frac{M_{x_{1}}^{\prime-}}{M_{x_{2}}^{\prime-}}\right\},
$$

where

$$
\begin{aligned}
& M_{x_{2}}^{\prime+}=\sup _{t>0, \mathbf{x} \in D^{+}, 0<\delta<k}\left\{x_{2} \mid f\left(t, x_{1}, x_{2}\right)+k=2 \delta\right\} \\
& M_{x_{2}}^{\prime-}=\inf _{t>0, \mathbf{x} \in D^{-}, 0<\delta<k}\left\{x_{2} \mid f\left(t, x_{1}, x_{2}\right)-k=-2 \delta\right\} \\
& 0<M_{x_{1}}^{\prime+} \leq M_{x_{1}}^{+}=\sup \left\{x_{1}>0 \mid\left\langle x_{1} x_{2}=M_{x_{2}}^{\prime+}\right\rangle \in D\right\} \\
& 0>M_{x_{1}}^{\prime-} \geq M_{x_{1}}^{-}=\inf \left\{x_{1}<0 \mid\left\langle x_{1} x_{2}=M_{x_{2}}^{\prime-}\right\rangle \in D\right\} \text {, }
\end{aligned}
$$

with the domain of attraction being

$$
\begin{gathered}
\mathbf{x}=\left[\begin{array}{ll}
x_{1} & x_{2}
\end{array}\right]^{T} \in D, \\
D^{+}=\left\{D \mid x_{1}>0, x_{2} \geq 0\right\} \\
D^{-}=\left\{D \mid x_{1}<0, x_{2} \leq 0\right\} .
\end{gathered}
$$

Proof. First, let us get an estimate for a bound on $x_{2}$ in the domain of attraction. For $0 \leq t_{0}<t_{1}<t_{2}$, without loss of generality, let us consider $0<x_{1}\left(t_{1}\right) \leq M_{x_{1}}^{+}, x_{1}\left(t_{2}\right)>x_{1}\left(t_{1}\right)>$ $0>-\beta=x_{1}\left(t_{0}\right), \beta \rightarrow 0$, and $t_{1}-t_{0}=h_{\max }<t_{2}-t_{0}$. Where $h_{\max }$ is the maximum delay such that the system remains in the domain of attraction, so that $h \leq h_{\max }$. Then, in the interval $t_{1}<t \leq t_{2}$, we have $\operatorname{sign}\left(x_{1}(t)\right)=\operatorname{sign}\left(x_{1}(t-\right.$ $h))=1$ and in the interval $t_{0}<t<t_{1}, \operatorname{sign}\left(x_{1}(t-h)\right)=$ $-\operatorname{sign}\left(x_{1}(t)\right)=-1$.

Therefore, with $h=h_{\max }$ and while $t_{0}<t<t_{1}$, we will have $x_{1}(t)>0$ and $x_{1}(t-h)<0$, so that $\dot{x}_{2}=f\left(t, x_{1}, x_{2}\right)+k$. Moreover, since $k-\left|f\left(t, x_{1}, x_{2}\right)\right| \geq c_{0}>0$, it can be seen that $x_{2}(t) \geq c_{0}\left(t-t_{0}\right)+x_{2}\left(t_{0}\right)$, and accordingly $x_{1}$ will grow while $\operatorname{sign}\left(x_{1}(t-h)\right)=-1$, but when $t>t_{1}$ and since $x_{1}>0$, we have $\operatorname{sign}\left(x_{1}(t-h)\right)=1$, and therefore $\dot{x}_{2}=f\left(t, x_{1}, x_{2}\right)-k$. Hence, $x_{2}(t) \leq-c_{0}\left(t-t_{1}\right)+x_{2}\left(t_{1}\right)$.

Therefore, there exists $t=t_{2}$, where $x_{2}\left(t_{2}\right)=0$, and accordingly when $t=t_{2} \leq t_{1}+x_{2}\left(t_{1}\right) / c_{0}, x_{1}$ arrives to a maximum and in the interval $t_{2}<t<t_{3}$, it will diminish until being zero at

$$
\begin{aligned}
t & =t_{3} \\
& \leq \frac{x_{2}\left(t_{1}\right)+\sqrt{x_{2}\left(t_{1}\right)^{2}+2 c_{0}\left[t_{1} x_{2}\left(t_{1}\right)+x_{1}\left(t_{1}\right)\right]}}{c_{0}}+t_{1} .
\end{aligned}
$$

This happens provided the system is in the domain of attraction, so the maximum value must occur for $\mathbf{x} \in D^{+}$. 
An estimate for the maximum $x_{2}$ reached value can be gotten noticing that $0<c_{0} \leq k$, since $k-\left|f\left(t, x_{1}, x_{2}\right)\right| \geq c_{0}>0$. Therefore, the biggest possible value for $\left|f\left(t, x_{1}, x_{2}\right)\right|$, inside the domain of attraction, occurs when $c_{0} \rightarrow 0$, making feasible that $\left|f\left(t, x_{1}, x_{2}\right)\right| \rightarrow k$. On the other hand, if $c_{0} \rightarrow$ $k,\left|f\left(t, x_{1}, x_{2}\right)\right|$ might only take the smallest possible value, $\left|f\left(t, x_{1}, x_{2}\right)\right| \rightarrow 0$. Hence, from the aforementioned, $0<$ $f\left(t, x_{1}, x_{2}\right)+k<2 k$, and the maximum value for $x_{2}, M_{x_{2}}^{\prime+}$ inside the domain of attraction may be estimated as

$$
M_{x_{2}}^{\prime+}=\sup _{t>0, \mathbf{x} \in D^{+}, 0<\delta<k}\left\{x_{2} \mid f\left(t, x_{1}, x_{2}\right)+k=2 \delta\right\} .
$$

Now, with a similar reasoning as above, it can be shown that once $x_{1}(t)<0$, a time will come such that $\operatorname{sign}\left(x_{1}(t-h)\right)=$ -1 , and if the dynamics are in the domain of attraction, $x_{1}$ will reach a minimum and begin moving toward the opposite direction remaining bounded. Therefore, an estimate for the minimum value of $x_{2}$ is

$$
M_{x_{2}}^{\prime-}=\inf _{t>0, \mathbf{x} \in D^{-}, 0<\delta<k}\left\{x_{2} \mid f\left(t, x_{1}, x_{2}\right)-k=-2 \delta\right\} .
$$

Now, since in the interval $t_{0} \leq t \leq t_{1}$ (see Figure 1), we have $\dot{x}_{1}=x_{2} \leq M_{x_{2}}^{\prime+}$, and considering that

$$
\begin{aligned}
0 & <M_{x_{1}}^{\prime+} \leq M_{x_{1}}^{+} \\
& =\sup \left\{x_{1}>0 \mid\left\langle x_{1} \quad x_{2}=M_{x_{2}}^{\prime+}\right\rangle \in D\right\},
\end{aligned}
$$

we have, $x_{1}\left(t_{1}\right) \leq M_{x_{1}}^{\prime+} \leq M_{x_{2}}^{\prime+} h_{\max }$. Therefore, it is clear that there exists $h^{\prime} \leq h_{\max }$ such that $M_{x_{1}}^{\prime+}=M_{x_{2}}^{\prime+} h^{\prime}$. And then for $h \leq h^{\prime}$, we have $h \leq M_{x_{1}}^{\prime+} / M_{x_{2}}^{\prime+}$. So that for this delay, the system dynamics remains in the domain of attraction for positive values of $x_{1}$.

Similarly, when $x_{1}<0, \dot{x}_{1}=x_{2} \geq M_{x_{2}}^{\prime-}$, and for $h_{\max }=$ $t_{4}-t_{3}$, where $t_{4}$ is the time when $x_{1}\left(t_{4}\right) \geq M_{x_{1}}^{\prime-} \geq M_{x_{2}}^{\prime-} h_{\max }$.

Considering $0>M_{x_{1}}^{\prime-} \geq M_{x_{1}}^{-}=\inf \left\{x_{1}<0\right.$ । $\left.\left\langle x_{1} \quad x_{2}=M_{x_{2}}^{\prime-}\right\rangle \in D\right\}$ and since $M_{x_{2}}^{\prime-}<0$, there exists $h^{\prime \prime} \leq h_{\max }$ such that $M_{x_{1}}^{\prime-}=M_{x_{2}}^{\prime-} h^{\prime \prime}$. Therefore, for $h \leq h^{\prime \prime}$, $h \leq M_{x_{1}}^{\prime-} / M_{x_{2}}^{\prime-}$, so that, for this delay, the system dynamics remains in the domain of attraction for negative values of $x_{1}$.

Hence, if

$$
h \leq \min \left\{\frac{M_{x_{1}}^{\prime+}}{M_{x_{2}}^{\prime+}}, \frac{M_{x_{1}}^{\prime-}}{M_{x_{2}}^{\prime-}}\right\},
$$

the system dynamics will stay inside the domain of attraction; so this is a sufficient condition in the delay size for amplitude bounded oscillations to exist.

Remark 4. For a second order sliding mode system in the form (1), with a specified delay, $h$, and noticing that the maximum $x_{1}$ amplitude is obtained when $\operatorname{sign}\left(x_{1}(t)\right)=$ $\operatorname{sign}\left(x_{1}(t-h)\right)$ and $x_{2}=0$, an estimate for the oscillation amplitude, $A_{x_{1}}$, can be obtained with

$$
\begin{aligned}
m_{x_{2}}^{\prime} h & \leq A_{x_{1}} \\
& \leq \max \left\{\begin{array}{l}
\sup _{t>0, \mathbf{x} \in D^{+}, 0<\delta<k}\left\{x_{1} \mid f\left(t, x_{1}, 0\right)-k=-2 \delta\right\}, \\
\sup _{t>0, \mathbf{x} \in D^{-}, 0<\delta<k}\left\{\left|x_{1}\right| \mid f\left(t, x_{1}, 0\right)+k=2 \delta\right\}
\end{array}\right\},
\end{aligned}
$$

where $m_{x_{2}}^{\prime}$ is the lowest magnitude value of $x_{2}$ for the interval when $\operatorname{sign}\left(x_{1}(t)\right)=-\operatorname{sign}\left(x_{1}(t-h)\right)$. Now, noticing that $\dot{x}_{2}=$ 0 is an extreme for $x_{2}$ in some cases, such lowest value for those cases may be obtained from

$$
\begin{aligned}
& m_{x_{2}}^{\prime} \\
& =\min \left\{\begin{array}{l}
\inf _{t>0, \mathbf{x} \in D^{+} \mid x_{1}(t-h)<0, x_{2}>0}\left\{x_{2} \mid f\left(t, x_{1}, x_{2}\right)+k=0\right\}, \\
\inf _{t>0, \mathbf{x} \in D^{-} \mid x_{1}(t-h)>0, x_{2}<0}\left\{\left|x_{2}\right| \mid f\left(t, x_{1}, x_{2}\right)-k=0\right\}
\end{array}\right\},
\end{aligned}
$$

verifying that $\ddot{x}_{2}>0$ when $\operatorname{sign}\left(x_{1}(t)\right)=1$ and $\ddot{x}_{2}<0$ when $\operatorname{sign}\left(x_{1}(t)\right)=-1$.

Remark 5. If the second order sliding mode system is of the form

$$
\begin{gathered}
\dot{x}_{1}=x_{2} \\
\vdots \\
\dot{x}_{n}=f\left(t, x_{1}, x_{2}\right)-k \operatorname{sign}\left(s\left(x_{1}(t-h), \ldots, x_{n-1}(t-h)\right)\right),
\end{gathered}
$$

such that

$$
\begin{gathered}
\dot{s}=\eta(\mathbf{x}) \\
\ddot{s}=\varphi(t, \mathbf{x})-m \operatorname{sign}(s(t-h)),
\end{gathered}
$$

so that $\mathbf{x}=0$ is stable for $h=0$, with the domain of attraction being $\mathbf{x} \in D$ and

$$
\begin{aligned}
& D^{s+}=\{D \mid s>0, \dot{s} \geq 0\}, \\
& D^{s-}=\{D \mid s<0, \dot{s} \leq 0\},
\end{aligned}
$$

an estimate of the maximum delay for steady oscillations in the sliding surface is

$$
h \leq \min \left\{\frac{M_{s}^{\prime+}}{M_{\dot{s}}^{\prime+}}, \frac{M_{s}^{\prime-}}{M_{\dot{s}}^{\prime-}}\right\},
$$

where

$$
\begin{gathered}
M_{\dot{s}}^{\prime+}=\sup _{t>0, \mathbf{x} \in D^{s+}, 0<\delta<m}\{\dot{s} \mid \varphi(t, \mathbf{x})+m=2 \delta\} \\
M_{\dot{s}}^{\prime-}=\inf _{t>0, \mathbf{x} \in D^{s-}, 0<\delta<m}\{\dot{s} \mid \varphi(t, \mathbf{x})-m=-2 \delta\} \\
0<M_{s}^{\prime+} \leq M_{s}^{+}=\sup \left\{s>0 \mid\left\langle s \quad \dot{s}=M_{\dot{s}}^{\prime+}\right\rangle \in D\right\} \\
0>M_{s}^{\prime-} \geq M_{s}^{-}=\inf \left\{s<0 \mid\left\langle s \quad \dot{s}=M_{\dot{s}}^{\prime-}\right\rangle \in D\right\} .
\end{gathered}
$$




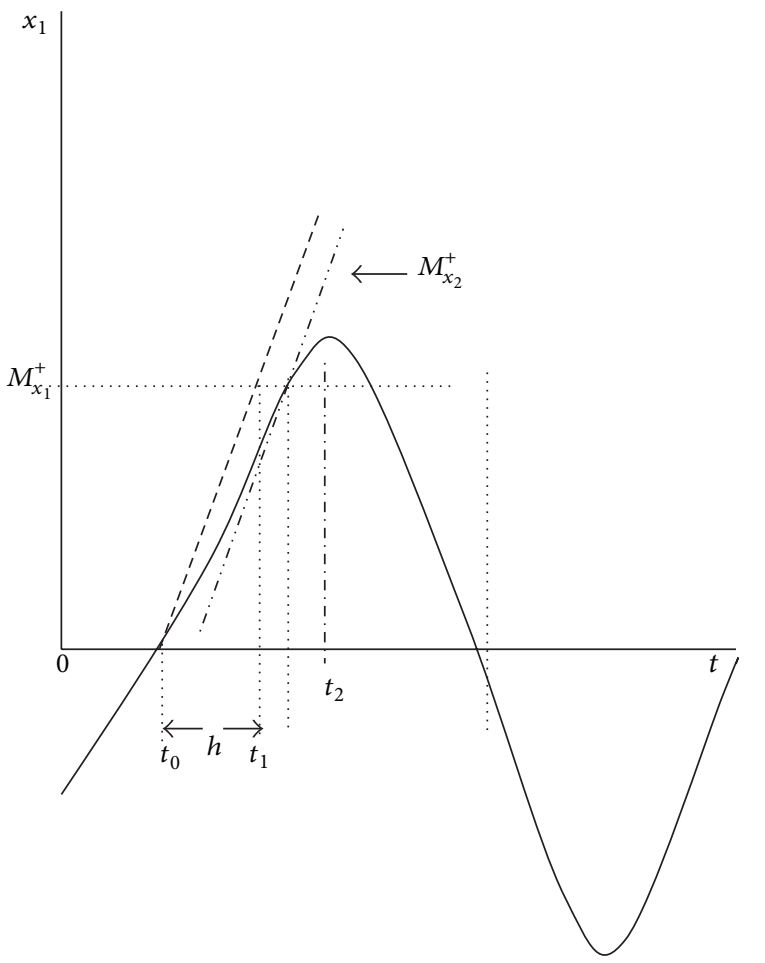

(a)

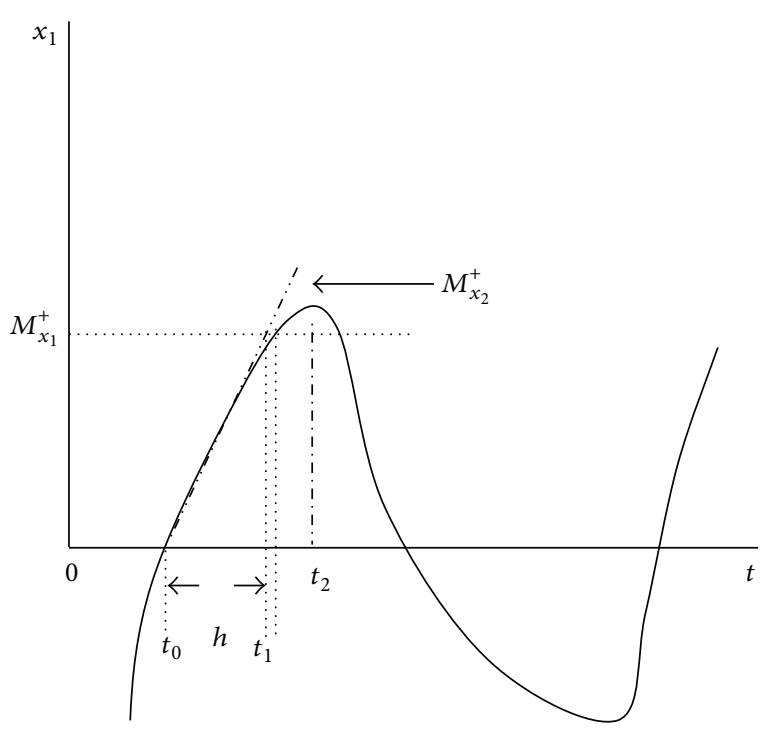

(b)

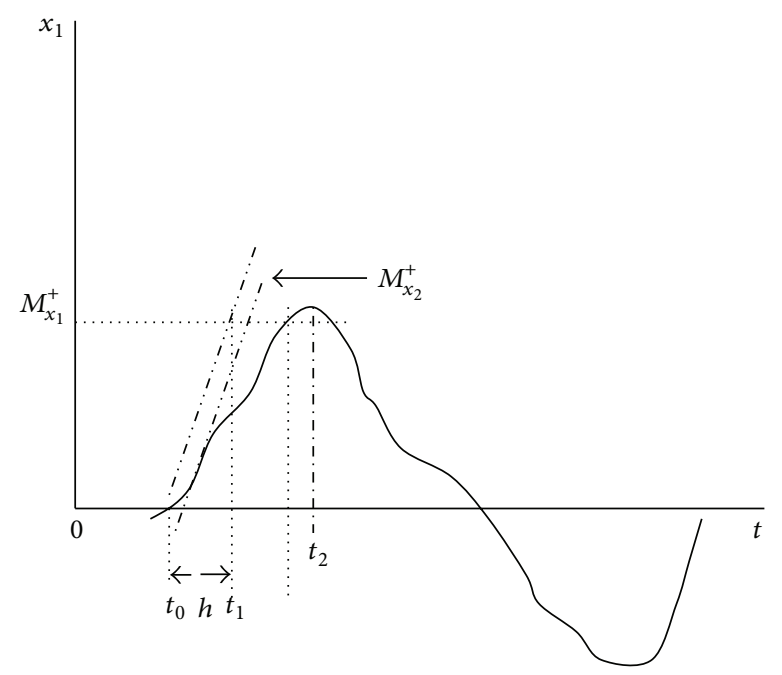

(c)

Figure 1: Behavior of $x_{1}$ for different forms of $f\left(t, x_{1}, x_{2}\right)$, where it can be seen as $h<t_{1}-t_{0}$ and maximum value for $x_{2}=M_{x_{2}}^{+}$, when $0<x_{1} \leq M_{x_{1}}^{+}$.

Remark 6. If the system is a first order sliding mode system with the form

$$
\begin{gathered}
\dot{x}_{1}=x_{2} \\
\vdots \\
\dot{x}_{n}=f\left(t, x_{1}, x_{2}\right)-k \operatorname{sign}\left(s\left(x_{1}(t-h), \ldots, x_{n}(t-h)\right)\right),
\end{gathered}
$$

such that

$$
\dot{s}=\psi(t, \mathbf{x})-p \operatorname{sign}(s(t-h)),
$$

so that $\mathbf{x}=0$ is stable for $h=0$, with the domain of attraction being $\mathbf{x} \in D$ and

$$
\begin{aligned}
D^{s+} & =\{D \mid s>0\} \\
D^{s-} & =\{D \mid s<0\},
\end{aligned}
$$


an estimate for the maximum delay where steady oscillations in the sliding surface are obtained may be gotten with

$$
h \leq \min \left\{\frac{M_{s}^{\prime+}}{M_{\dot{s}}^{\prime+}}, \frac{M_{s}^{\prime-}}{M_{\dot{s}}^{\prime-}}\right\},
$$

where

$$
\begin{gathered}
M_{\dot{s}}^{\prime+}=\sup _{t>0, \mathbf{x} \in D^{s+}, 0<\delta<p}\{\dot{s} \mid \psi(t, \mathbf{x})+p \leq 2 \delta\} \\
M_{\dot{s}}^{\prime-}=\inf _{t>0, \mathbf{x} \in D^{s-}, 0<\delta<p}\{\dot{s} \mid \psi(t, \mathbf{x})-p \geq-2 \delta\} \\
0<M_{s}^{\prime+} \leq M_{s}^{+}=\sup \left\{s>0 \mid \dot{s}=M_{\dot{s}}^{\prime+} \in D\right\} \\
0>M_{s}^{\prime-} \geq M_{s}^{-}=\inf \left\{s<0 \mid \dot{s}=M_{\dot{s}}^{\prime+} \in D\right\} .
\end{gathered}
$$

Remark 7. For a first order sliding mode system with amplitude bounded oscillations, if an upper bound for the sliding surface derivative magnitude is known (see Remark 6), together with the delay, $h$, an upper bound for the oscillation amplitude, $A_{s}$, of the sliding surface, $s$, can be obtained with

$$
A_{s} \leq \max \left\{M_{\dot{s}}^{\prime+} h,\left|M_{\dot{s}}^{\prime-}\right| h\right\} .
$$

\section{Examples}

In this section, three examples are given to validate the results presented above.

4.1. Reaching Equilibrium Point in Finite Time for Second Order Sliding Modes. Let us consider the following system:

$$
\begin{aligned}
& \dot{x_{1}}=x_{2} \\
& \dot{x_{2}}=x_{1}-5 x_{2}-5 \operatorname{sign}\left(x_{1}\right) \text {. }
\end{aligned}
$$

It is easy to verify that system dynamics are unstable if $5 \operatorname{sign}\left(x_{1}\right)$ is not present. In order to verify if the dynamics of (43) reach in finite time the equilibrium point in zero, Proposition 1 is used. Let's consider $x_{1}>0$. Since $k=5$, $\dot{x}_{2}>-k=-5$ must be complied and also $\dot{x}_{2} \leq-c_{0}$, such that $x_{2}>-5 t+x_{2}(0)$, and $x_{1} \leq-c_{0} t^{2} / 2+x_{2}(0) t+x_{1}(0)$.

Therefore, $\dot{x}_{2} \leq-c_{0} t^{2} / 2+\left(25+x_{2}(0)\right) t+x_{1}(0)-5 x_{2}(0)-5$. From this expression, it can be observed that the time when the biggest value for $\dot{x}_{2}$ (less negative) is $t=0$, since for $\left|f\left(t, x_{1}, x_{2}\right)\right|<5$, it is required that $\left|x_{2}\right|<5$, then from $t=0, \dot{x}_{2}$ goes more negative while $x_{1}>0$. Similarly, it can be shown that if the initial condition is $x_{1}<0$, the lowest value for $\dot{x}_{2}$ occurs at $t=0$, such that $k-\left|f\left(t, x_{1}, x_{2}\right)\right| \geq c_{0}$. Hence, a sure way to choose the initial conditions would be $\left|x_{1}(0)-5 x_{2}(0)\right|<5$.

So that, for this example, we choose $x_{1}(0)=4, x_{2}(0)=0$. Therefore, at $t=0,\left|f\left(t, x_{1}, x_{2}\right)\right|<5$, and just afterwards, $x_{2}$ is and remains negative while $x_{1}>0$. Therefore $x_{1}$ goes down until reaching the first zero crossing. Knowing that

$$
x_{1}(t)=-.9642 e^{0.19258 t}-0.03328 e^{-5.1958 t}+5 \text {, }
$$

it is easy to see that $x_{1}=0$, when $x_{2}=-.93$.
Up to now, first conditions for the Proposition 1 are met in order to get the first zero crossing. Now, to see if the condition for finite time surface reaching is met, let us consider an arbitrary time of zero crossing from positive to negative $t_{n}$. In the interval $t_{n} \leq t \leq t_{n+1}$, the maximum value for $\left|f\left(t, x_{1}, x_{2}\right)\right|$ is at $t_{n}$, since this is the initial time in this interval. Then $f\left(t_{n}, 0, c_{n}\right)=-5 c_{n}$ and at $t=t_{n+1}, f\left(t_{n+1}, 0, c_{n+1}\right)=-5 c_{n+1}$. From Proposition 1, it is needed

$$
\frac{\left|c_{n}\right|}{\left|c_{n+1}\right|}>\frac{\left(k-\left|f\left(t_{n}, 0, c_{n}\right)\right|\right)}{\left(k-\left|f\left(t_{n+1}, 0, c_{n+1}\right)\right|\right)}=\frac{5-5\left|c_{n}\right|}{5-5\left|c_{n+1}\right|} \text {. }
$$

Resulting in the condition $\left|c_{n}\right|>\left|c_{n+1}\right|$.

Let's see if the system meets such a condition. Meanwhile, $x_{1}<0$ in the interval $t_{n} \leq t \leq t_{n+1}$,

$$
\begin{gathered}
x_{1}(t)=A e^{0.19258\left(t-t_{n}\right)}+B e^{-5.1958\left(t-t_{n}\right)}-5 \\
x_{2}(t)=0.19258 A e^{0.19258\left(t-t_{n}\right)}-5.1958 B e^{-5.1958\left(t-t_{n}\right)},
\end{gathered}
$$

with

$$
\begin{aligned}
& A=\frac{5-0.1958 c_{n}}{1.037}, \\
& B=\frac{5+5.1958 c_{n}}{27.963} .
\end{aligned}
$$

Now, considering that in the following zero crossing, $t=t_{n+1}$,

$$
\begin{gathered}
0=A e^{0.19258\left(t_{n+1}-t_{n}\right)}+B e^{-5.1958\left(t_{n+1}-t_{n}\right)}-5 \\
c_{n+1}=0.19258 A e^{0.19258\left(t_{n+1}-t_{n}\right)}-5.1958 B e^{-5.1958\left(t_{n+1}-t_{n}\right)},
\end{gathered}
$$

and solving (48), using expressions for $A$ and $B$ in (46),

$$
c_{n+1}=0.963-0.963 e^{-5.1958\left(t_{n+1}-t_{n}\right)}-e^{-5.1958\left(t_{n+1}-t_{n}\right)} c_{n} .
$$

This is an asymptotically stable first order difference equation for $c_{n}$ dynamics, since $t_{n+1}>t_{n}$, giving the eigenvalue magnitude less than one and guaranteeing that $\left|c_{n+1}\right|<\left|c_{n}\right|$, complying with the condition for reaching the surface in finite time. Eventually, when $t_{n+1}=t_{n}$, the equilibrium point is reached, $c_{n}=0$. Therefore, system dynamics are stable and reach the surface in finite time without need of using a measure or estimate of $x_{2}$ for the control signal. In Figure 2, the behavior of $x_{1}$ and $x_{2}$ is shown using $x_{1}(0)=4, x_{2}(0)=0$. It is seen that effectively, $x_{1}$ approaches smoothly and in finite time to zero and chattering is confined to $x_{2}$, which also approaches in finite time to zero.

4.2. Maximum Permissible Delay in the Second Order Sliding Mode System. Knowing that sliding modes are gotten for system (43), now a delay in the control is introduced as shown below:

$$
\begin{gathered}
\dot{x}_{1}=x_{2} \\
\dot{x}_{2}=x_{1}-5 x_{2}-5 \operatorname{sign}\left(x_{1}(t-h)\right) .
\end{gathered}
$$




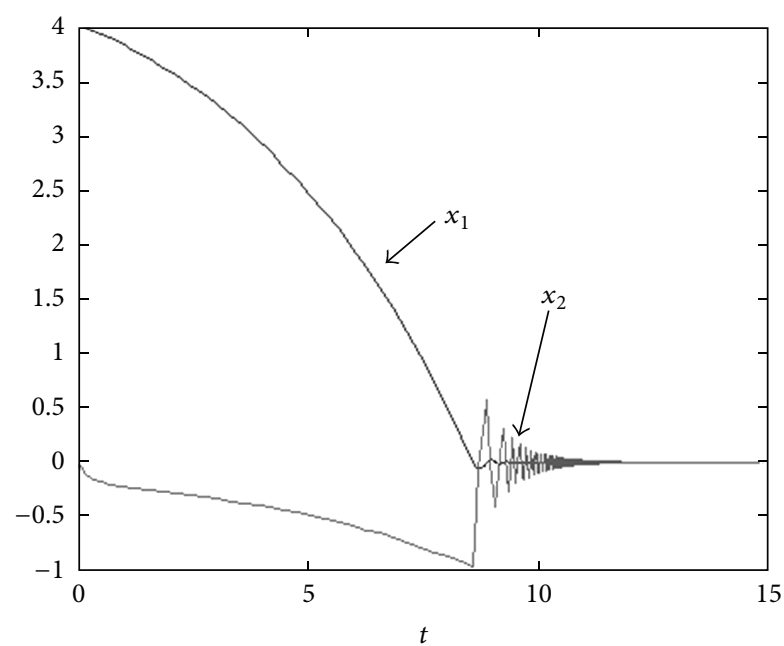

FIGURE 2: Behavior of system (43) dynamics showing that $x_{1}=0$ and $x_{2}=0$ are reached in finite time. As expected for second order sliding mode, chattering is not present in $x_{1}$.

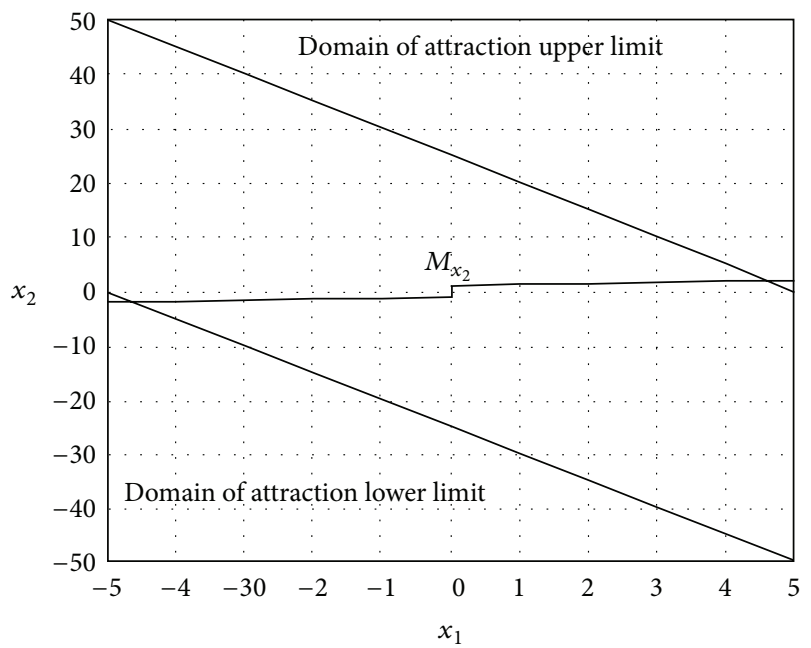

FIGURE 3: Estimate for biggest value of $x_{2}$ in the system with delay (50) inside the domain of attraction.

Now, using (22), $M_{x_{1}}^{\prime}-5 M_{x_{2}}^{\prime}+5=2 \delta$, which gives the maximum with $\delta=0$

$$
M_{x_{2}}^{\prime} \leq \frac{M_{x_{1}}^{\prime}+5}{5} .
$$

The domain of attraction is shown in Figure 3, together with all possible values for $M_{x_{2}}^{\prime}$. It is noticed that at $x_{1}=4.5, M_{x_{2}}^{\prime}=$ 1.9 intersects the upper limit of the domain of attraction. Therefore

$$
h<\frac{M_{x_{1}}^{\prime}}{M_{x_{2}}^{\prime}}=\frac{4.5}{1.9}=2.368 .
$$

In this example, $h=2.36$ was used along with initial conditions set to $x_{1}=2$ and $x_{2}=0$, in order to ensure the initial crossing by zero since for $t \in[0, h]$ no control is available. To estimate the oscillation amplitude, Remark 4 of

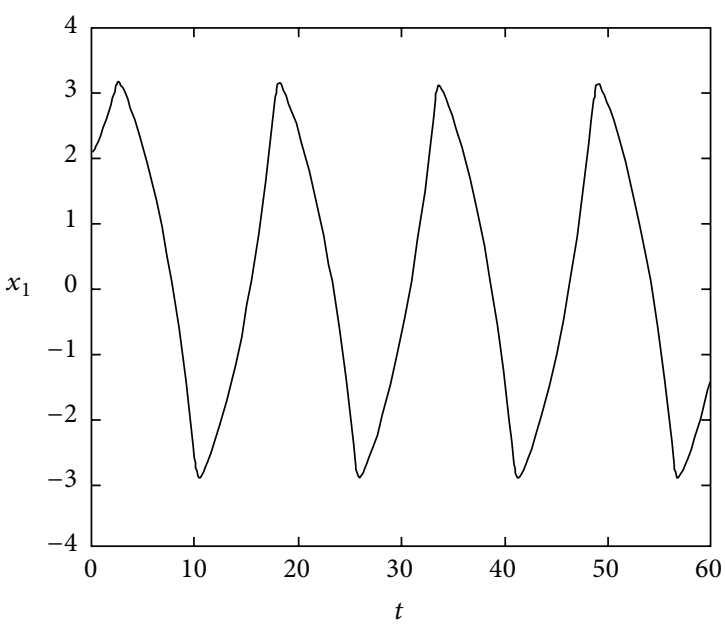

FIGURE 4: Behavior of dynamics for system (50) with delay $h=2.36$.

Proposition 3 is used. To obtain $m_{x_{2}}^{\prime}$, we have $x_{1}-5 x_{2}+5=0$. And so $m_{x_{2}}^{\prime}=\min _{x_{1}>0}\left(\left(5+x_{1}\right) / 5\right)=1$. Therefore, $A_{x_{1}} \geq$ $m_{x_{2}}^{\prime} h=2.36$. Now, the biggest value for $x_{1}$ is obtained from $x_{1}-5=-2 \delta$, so that $A_{x_{1}} \leq \max _{0 \leq \delta<5}(5-2 \delta)=5$.

Hence, the oscillation amplitude for $x_{1}$ is bounded with a value between 2.36 and 5. In Figure 4, it can be verified that $h=2.36$ is a sufficient condition for bounded oscillations to occur. Also, the amplitude is seen to be a little bigger than 3, which is inside the estimated limits. For this case the describing function approach cannot be used to estimate the amplitude, because of the instability of the associated linear system.

4.3. Amplitude Estimate for a Globally Stable System. Considering a case where the equilibrium point of the involved system is globally stable such as

$$
\begin{gathered}
\dot{x}_{1}=x_{2}, \\
\dot{x}_{2}=-5 x_{2}-5 \operatorname{sign}\left(x_{1}(t-h)\right) .
\end{gathered}
$$

Using Remark 4 of Proposition 3, the inferior bound for amplitude $A_{x_{1}}$ with $m_{x_{2}}^{\prime}=5 / 5=1$ is $A_{x_{1}}>h m_{x_{2}}^{\prime}=h$. If $h=5$, then $A>5$. In Figure 5, the system response for $x_{1}$ is seen. The oscillation amplitude is a little bigger than 5 , verifying the estimated value. Also, since $x_{2}$ is almost constant, the estimated amplitude is very close to the real one. If the describing function approach was used, the estimated amplitude would be around 4.44, which is lower than the minimum estimated with the proposed method. In this particular case, an estimate of the superior bound for $x_{1}$ using Remark 4 cannot be obtained, since $x_{1}$ is not explicit in $f(t, x, 0)-k=-2 \delta$.

\section{Conclusions}

Stability analysis for a class of second order sliding mode systems, which does not need measuring or estimation of the derivative of the sliding variable for the control signal, 


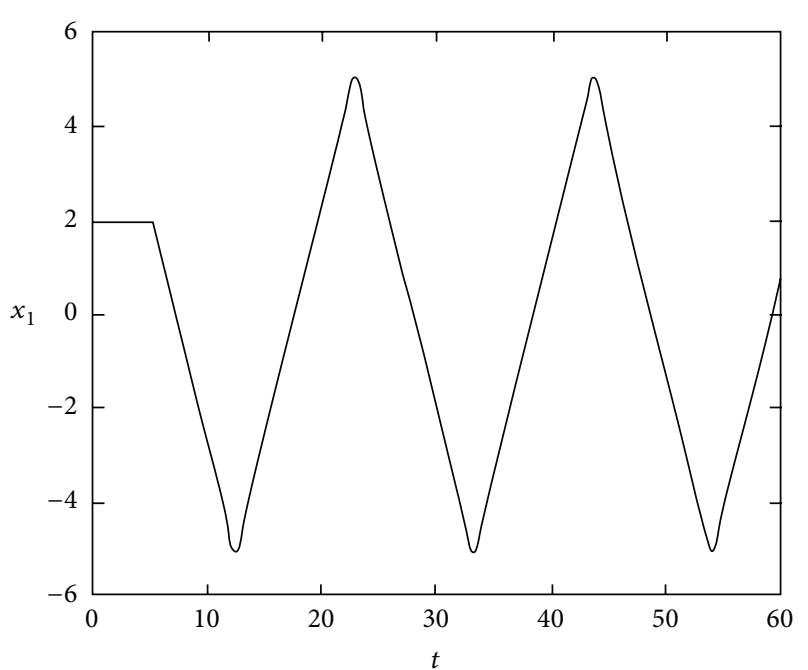

FIGURE 5: Behavior of globally stable system (53) with a delay $h=5$.

was presented. Also, when delay in input is present, bounded oscillations are studied. The system can be linear or nonlinear. The class of systems treated is a more general class of the systems studied in [20], where sufficient conditions are given for stationary oscillations to exist using another approach and no means is given to estimate amplitude of oscillations. The analysis presented in this paper is based on two propositions. One of them gives sufficient conditions for sliding mode existence for a class of second order sliding mode systems. The other can be used as a method based on maximum derivative to get a delay estimate on the control signal for a class of second order sliding mode systems, giving sufficient conditions to maintain amplitude bounded oscillations on the sliding surface. Also, for a given delay in input, oscillation amplitude for the sliding surface dynamics can be estimated if the system state is in the attraction region. On the other hand, when oscillation amplitude is known, such as hysteresis happening in the discontinuous input, frequency of oscillation can be estimated. Numerical examples for second order sliding modes were presented to illustrate the proposed approach, first two examples have unstable dynamics when no input is applied. Third example has global stability for the considered input. It is clear that very conservative results using this method would arise for systems where the expected sliding surface derivative is much lower than the maximum derivative. For linear stable processes, the describing function method has been used in the literature to estimate oscillations amplitude. In the second example such approach cannot be used since the linear process is unstable. In the third example, the simulation shows the amplitude just a little bigger than the minimum estimated by the approach presented here. For this example, the describing function method gives a lower estimate than the minimum given by the proposed approach.

\section{References}

[1] A. Levant, "Sliding order and sliding accuracy in sliding mode control," International Journal of Control, vol. 58, no. 6, pp. 12471263, 1993.
[2] G. Bartolini, A. Ferrara, and E. Usai, "Chattering avoidance by second-order sliding mode control," IEEE Transactions on Automatic Control, vol. 43, no. 2, pp. 241-246, 1998.

[3] A. Levant, "Robust exact differentiation via sliding mode technique," Automatica, vol. 34, no. 3, pp. 379-384, 1998.

[4] A. Levant, "Homogeneity approach to high-order sliding mode design," Automatica, vol. 41, no. 5, pp. 823-830, 2005.

[5] H. An, J. Li, J. Wang, J. Wang, and H. Ma, "Second-order geometric sliding mode attitude observer with application to quadrotor on a test bench," Mathematical Problems in Engineering, vol. 2013, Article ID 328974, 12 pages, 2013.

[6] M. Defoort, T. Floquet, A. Kokosy, and W. Perruquetti, "A novel higher order sliding mode control scheme," Systems \& Control Letters, vol. 58, no. 2, pp. 102-108, 2009.

[7] N. Zhang, M. Yang, Y. Jing, and S. Zhang, "Congestion control for diffserv network using second-order sliding mode control," IEEE Transactions on Industrial Electronics, vol. 56, no. 9, pp. 3330-3336, 2009.

[8] B. Beltran, M. E. H. Benbouzid, and T. Ahmed-Ali, "Secondorder sliding mode control of a doubly fed induction feneratordriven wind turbine," IEEE Transactions on Energy Conversion, vol. 27, no. 2, pp. 261-269, 2012.

[9] M. Akian, P.-A. Bliman, and M. Sorine, "Control of delay systems with relay," Journal of Mathematical Control and Information, vol. 19, no. 1-2, pp. 133-155, 2002.

[10] F. Gouaisbaut, M. Dambrine, and J. P. Richard, "Robust control of delay systems: a sliding mode control design via LMI," Systems \& Control Letters, vol. 46, no. 4, pp. 219-230, 2002.

[11] X. Li and S. Yurkovich, "Sliding mode control of delayed systems with application to engine idle speed control," IEEE Transactions on Control Systems Technology, vol. 9, no. 6, pp. 802-810, 2001.

[12] J.-P. Richard, “Time-delay systems: an overview of some recent advances and open problems," Automatica, vol. 39, no. 10, pp. 1667-1694, 2003.

[13] Y. Xia and Y. Jia, "Robust sliding-mode control for uncertain time-delay systems: an LMI approach," IEEE Transactions on Automatic Control, vol. 48, no. 6, pp. 1086-1092, 2003.

[14] M. Basin, J. Rodriguez-Gonzalez, L. Fridman, and P. Acosta, "Integral sliding mode design for robust filtering and control of linear stochastic time-delay systems," International Journal of Robust and Nonlinear Control, vol. 15, no. 9, pp. 407-421, 2005.

[15] L. Wu, X. Su, and P. Shi, "Sliding mode control with bounded $L_{2}$ gain performance of Markovian jump singular time-delay systems," Automatica, vol. 48, no. 8, pp. 1929-1933, 2012.

[16] Y. Xia, G.-P. Liu, P. Shi, J. Chen, and D. Rees, "Robust delaydependent sliding mode control for uncertain time-delay systems," International Journal of Robust and Nonlinear Control, vol. 18, no. 11, pp. 1142-1161, 2008.

[17] E. Fridman, L. Fridman, and E. Shustin, "Steady modes in relay control systems with time delay and periodic disturbances," Journal of Dynamic Systems, Measurement and Control, Transactions of the ASME, vol. 122, no. 4, pp. 732-737, 2000.

[18] I. Boiko, "Asymmetric limit cycles and transfer properties of relay servo systems with integrating plants," in Proceedings of the 41st IEEE Conference on Decision and Control, pp. 3932-3936, Las Vegas, Nev, USA, December 2002.

[19] P. Acosta, A. Polyakov, L. Fridman, and V. Strygin, "Estimation of amplitude of oscillations in sliding mode systems caused by time delay," Asian Journal of Control, vol. 6, no. 4, pp. 507-513, 2004. 
[20] E. Shustin, E. Fridman, and L. Fridman, "Oscillations in a second-order discontinuous system with delay," Discrete and Continuous Dynamical Systems A, vol. 9, no. 2, pp. 339-358, 2003.

[21] L. Levaggi and E. Punta, "Analysis of a second-order slidingmode algorithm in presence of input delays," IEEE Transactions on Automatic Control, vol. 51, no. 8, pp. 1325-1332, 2006. 


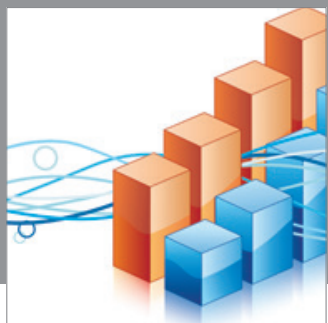

Advances in

Operations Research

mansans

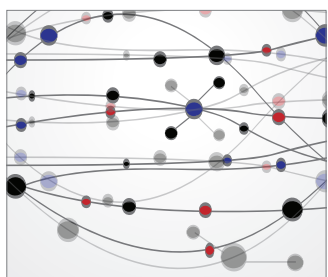

The Scientific World Journal
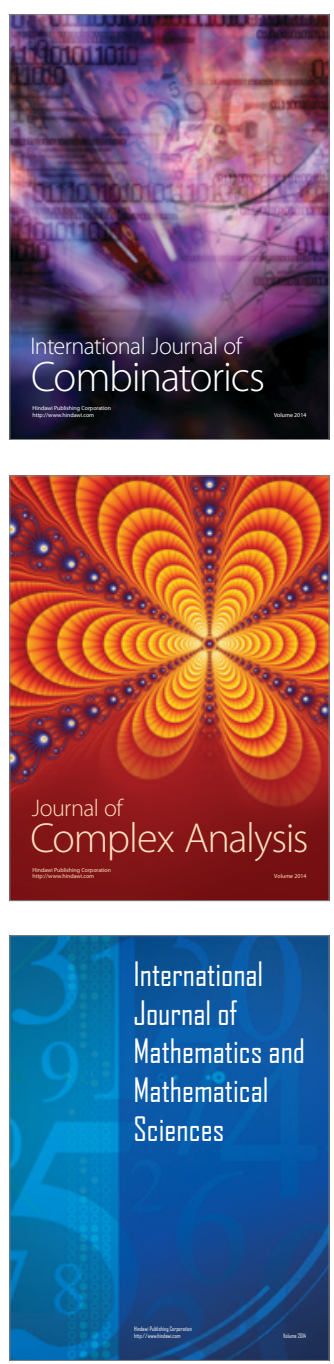
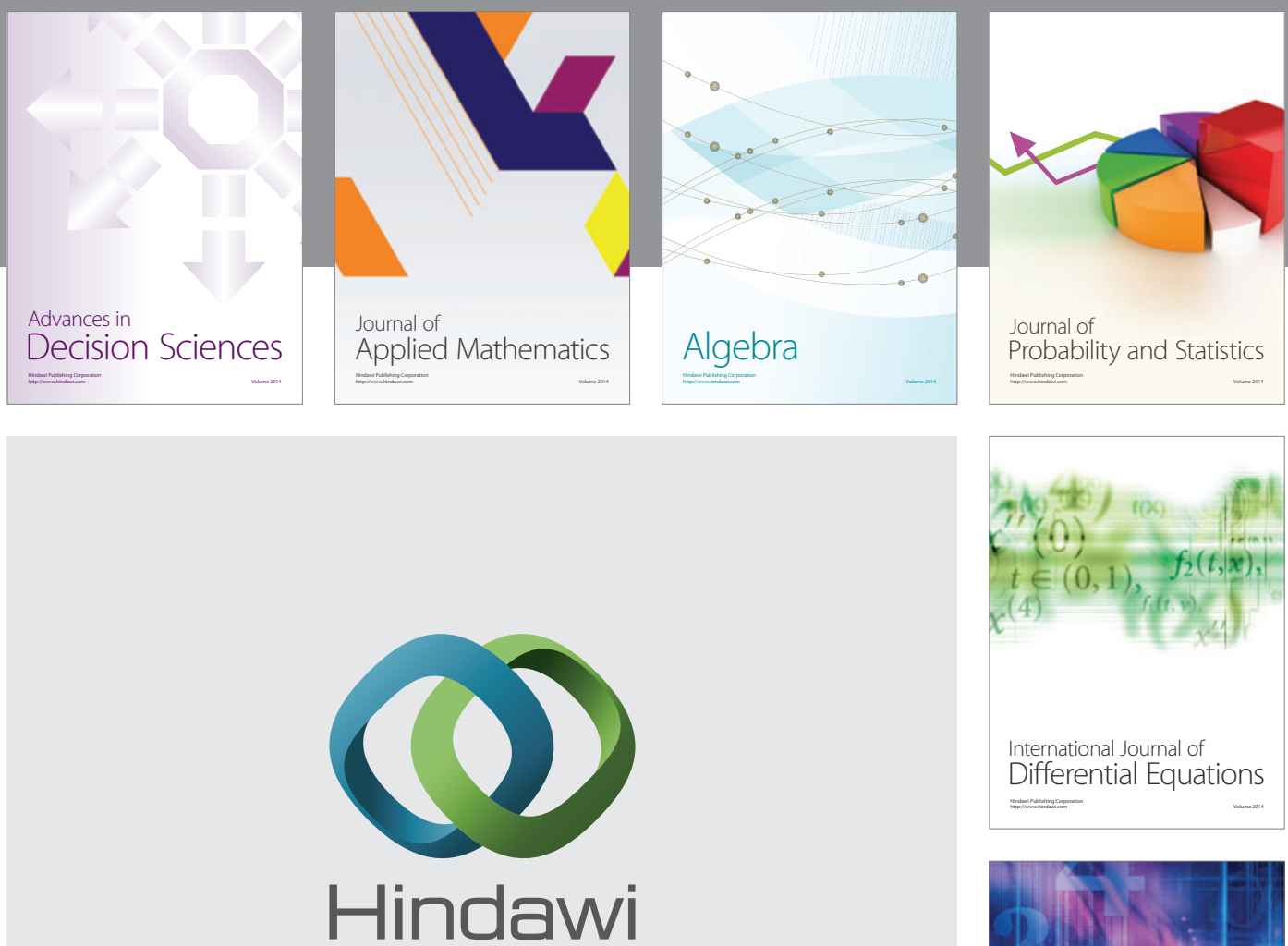

Submit your manuscripts at http://www.hindawi.com
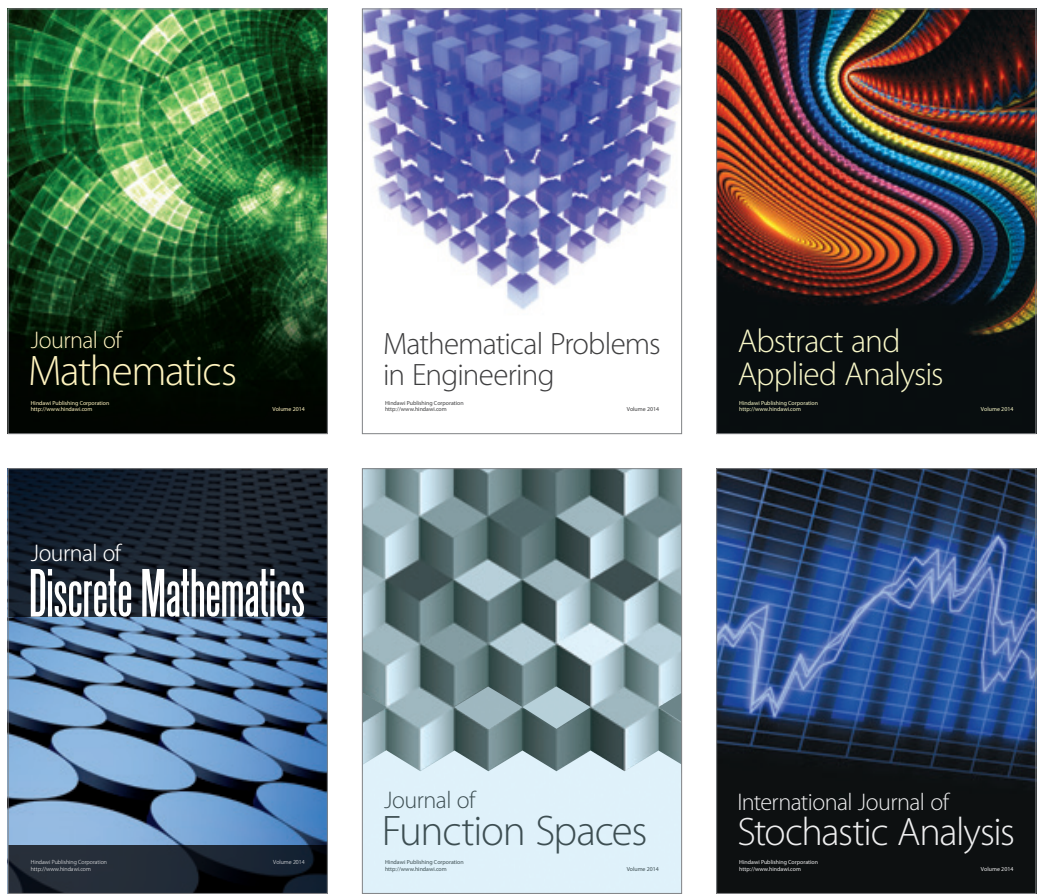

Journal of

Function Spaces

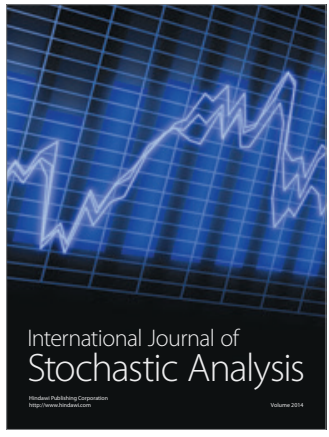

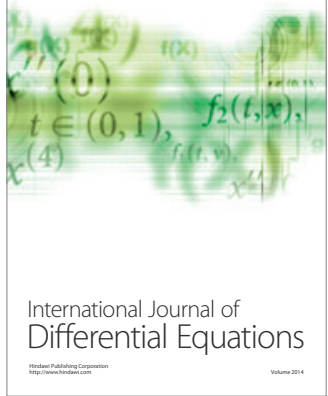
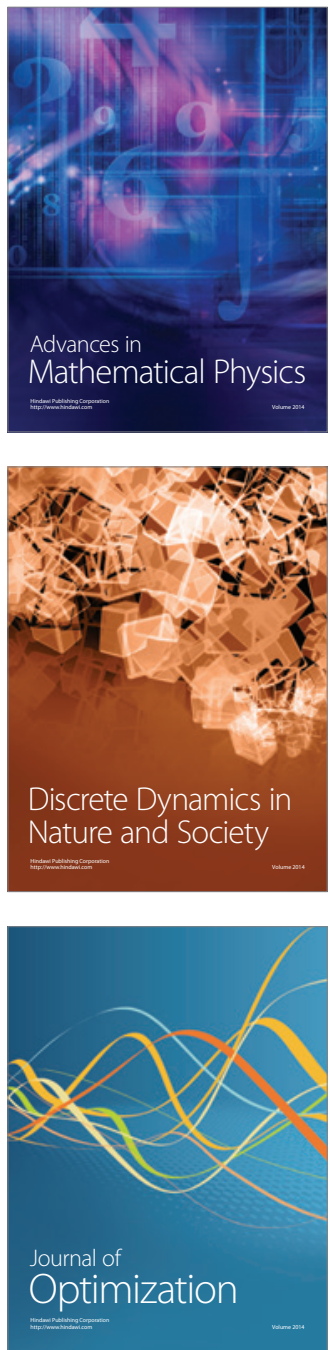